Proyecciones Journal of Mathematics

Vol. 37, No 2, pp. 181-198, June 2018.

Universidad Católica del Norte

Antofagasta - Chile

\title{
Super vertex mean labeling of cycles through different ways
}

\author{
A. Lourdusamy \\ St. Xavierś College (Autonomous), India \\ and \\ Sherry George \\ St. Xavierś College (Autonomous), India \\ Received: April 2016. Accepted : November 2017
}

\begin{abstract}
A super vertex mean labeling $f$ of a $(p, q)$ - graph $G=(V, E)$ is defined as an injection from $E$ to the set $\{1,2,3, \cdots, p+q\}$ that induces for each vertex $v$ the label defined by the rule $f^{v}(v)=$ Round $\left(\frac{\sum_{e \in E_{v}} f(e)}{d(v)}\right)$, where $E_{v}$ denotes the set of edges in $G$ that are incident at the vertex $v$, such that the set of all edge labels and the induced vertex labels is $\{1,2,3, \cdots, p+q\}$. In this paper, we investigate the super vertex mean labeling behavior of cycles by giving various ways by which they can be labeled.
\end{abstract}

Subjclass : Primary 05C38, 05C78.

Keywords : Super Vertex Mean label, Cycles, types of labeling. 


\section{Introduction}

A graph is an ordered pair $(V(G), E(G))$, consisting of a finite non empty set $V(G)$ of objects called points or vertices and a set $E(G)$ of 2-element subsets of $V(G)$, known as edges. The sets $V(G)$ and $E(G)$ are the vertex set and edge set respectively. The cardinality of $V(G)$ is the order of the graph $G$ and is often denoted by $|V(G)|=p$, and that of $E(G)$ is the size of $G$ and is denoted by $|E(G)|=q$. A graph of order $p$ and size $q$ is often called a $(p, q)$ - graph.

A labeling of a graph $G$ is an assignment of labels either to the vertices or edges. Harmonious labeling is one of the fundamental labeling introduced by Graham and Solane [4] in 1980 in connection with their study on error correction code. Mean labeling was introduced by Somasundaram and Ponraj [13]. Let $G=(V, E)$ be a simple graph with $p$ vertices and $q$ edges. A mean labeling $f$ is an injection from $V$ to the set $\{0,1,2, \cdots, q\}$ that induces for each edge $u v$ the label $\left[\frac{f(u)+f(v)}{2}\right]$ such that the set of edge labels is $\{1,2, \cdots, q\}$. A graph that accepts a mean labeling is known as mean graph.

A super mean labeling $f$ is an injection from $V$ to the set $\{1,2, \cdots, p+q\}$ [10] that induces for each edge $u v$ the label $\left\lceil\frac{f(u)+f(v)}{2}\right\rceil$ such that the set of all vertex labels and the induced edge labels is $\{1,2, \cdots, p+q\}$. Some results on mean labeling and super mean labeling can be found in [6], [8], [9], [10] and [14].

Lourdusamy and Seenivasan [5] introduced vertex mean labeling as an edge analogue of mean labeling. A graph that has a vertex mean labeling is called a vertex mean graph or $V$ - mean graph.

Lourdusamy et al. [7] brought in a new type of mean labeling, called Super vertex mean labeling of graphs.

\section{Super Vertex Mean Labeling}

Definition 2.1. [7] A super vertex mean labeling $f$ of a $(p, q)$ - graph $G=(V, E)$ is defined as an injection from $E$ to the set $\{1,2,3, \cdots, p+q\}$ that induces for each vertex $v$ the label defined by the rule $f^{v}(v)=$ Round $\left(\frac{\sum_{e \in E_{v}} f(e)}{d(v)}\right)$, where $E_{v}$ denotes the set of edges in $G$ that are incident at the vertex $v$, such that the set of all edge labels and the induced vertex labels is $\{1,2,3, \cdots, p+q\}$.

A graph that accepts super vertex mean labeling is called a Super Ver- 
tex Mean, that is SVM - graph in short.

Observations 2.1: A graph having isolated vertices or leaves cannot be an SVM - graph. For, if $\operatorname{deg}(v)=0$ for any vertex $v$ of $G$, the above definition is not defined and if $\operatorname{deg}(v)=1$ for any vertex $v$ of $G$, the induced vertex label remains the same as the label of the edge that is incident on the vertex $v$. Therefore, for a graph to be SVM - graph it is necessary that $\operatorname{deg}(v) \geq 2$ for all vertices $v$ in $V(G)$. It is obvious that no tree is a SVM graph.

\section{Super Vertex Mean Labeling of Cycles}

Theorem 3.1. All the cycles except $C_{4}$ are $S V M$ - graphs.

Proof. Illustration: For $C_{4}$, we have $p=4$ and $q=4$.

$f(E) \cup f(V)=\{1,2,3,4,5,6,7,8\}=\{1,2,3, \cdots, p+q\}$.

It is obvious that 1 and 8 cannot be induced vertex labels, so necessarily belong to $f(E)$. Since 2 cannot be an edge label, it belongs to $f(V)$ and for 2 to be a vertex label, it has to labeled on a vertex on which the edges that are labeled 1 and 3 lie. And so, 3 also belongs to $f(E)$.

Therefore, 8 can be labeled on an edge that is adjacent to an edge labeled 3 or 1 . The following cases arise:

Case 1: Let 8 be labeled on an edge adjacent to the edge labeled 3 .

Now, 7 cannot be labeled on any edges. The remaining options are that, we label either 4 or 5 on the fourth edge.

Case 1(a): Let 4 be labeled on the fourth edge. This is not an SVM labeling as the vertices that are incident on the edge labeled 8 get the same induced label 6 .

Case 1(b): Let 5 be labeled on the fourth edge. This also is ruled out as one of the vertices incident on the edge labeled 5 gets the label 3 , which is contrary to the assumption that 3 has to be an edge label.

Therefore, Case 1 is not possible.

Case 2: Let 8 be labeled on an edge which is adjacent to the edge labeled 1 .

In this case 7 cannot be an edge label and if 7 is an induced vertex label, then one of the induced vertex labels gets repeated. Therefore, Case 2 also is impossible. 
So, we conclude that the cycle $C_{4}$ is not a SVM - graph.

Now we prove that $C_{n}, n \geq 5$ is a SVM - graph. There can be two cases depending upon whether $n$ is odd or even.

Case 3: $n \equiv 1(\bmod 2)$. Let $C_{n}$ be an odd cycle with $n$ vertices. Let $\left\{e_{1}, e_{2}, \cdots, e_{n}\right\}$ be the edge set and $\left\{v_{1}, v_{2}, \cdots, v_{n}\right\}$ be the vertex set of $C_{n}$, such that $e_{i}=v_{i} v_{i+1}, 1 \leq i \leq n-1$ and $e_{n}=v_{n} v_{1}$.

Let $n=2 r+1$. The edges of $C_{n}$ are labeled as follows:

$$
f\left(e_{i}\right)= \begin{cases}2 i-1 & \text { if } 1 \leq i \leq r+1 \\ 2 i & \text { if } r+2 \leq i \leq n\end{cases}
$$

It is easy to observe that $f$ is injective. The induced vertex labels are given as follows:

$$
f^{v}\left(v_{i}\right)= \begin{cases}n+1 & \text { if } i=1 \\ 2 i-2 & \text { if } 2 \leq i \leq r+1 \\ 2 i-1 & \text { if } r+2 \leq i \leq n\end{cases}
$$

It is clear that,

$$
\begin{aligned}
& f(E) \cup f^{v}(V) \\
& =\{1,3,5, \cdots, 2 r+1,2 r+4,2 r+6, \cdots, 2 n-2,2 n\} \cup \\
& \{2 r+2=n+1,2,4, \cdots, 2 r-2,2 r, 2 r+3,2 r+5, \cdots, 2 n-3,2 n-1\} \\
& =\{1,3, \cdots, 2 r+1=n, 2 r+3,2 r+5, \ldots, 2 n-1\} \cup \\
& \{2,4, \cdots, 2 r=n-1, n+1=2 r+2,2 r+4,2 r+6, \cdots, 2 n-1,2 n\} \\
& =\{2 i-1: 1 \leq i \leq n\} \cup\{2 i: 1 \leq i \leq n\} \\
& =\{1,2,3, \cdots, 2 n\}
\end{aligned}
$$

Case 4: $n \equiv 0(\bmod 2)$

Let $\left\{e_{1}, e_{2}, \cdots, e_{n}\right\}$ be the edge set and $\left\{v_{1}, v_{2}, \cdots, v_{n}\right\}$ be the vertex set of $C_{n}$ such that $e_{i}=v_{i} v_{i+1}, 1 \leq i \leq n-1$ and $e_{n}=v_{n} v_{1}$.

Let $n=2 r$. 
The edges of $C_{n}$ are labeled as follows:

$$
f\left(e_{i}\right)= \begin{cases}1 & \text { if } i=1 \\ 3 & \text { if } i=2 \\ 7 & \text { if } i=3 \\ 4 i-4 & \text { if } 4 \leq i \leq r+1 \\ 4 n-4 i+5 & \text { if } r+2 \leq i \leq n-1 \\ 6 & \text { if } i=n\end{cases}
$$

It is easy to observe that $f$ is injective. The induced vertex labels are given as follows:

$$
f^{v}\left(v_{i}\right)= \begin{cases}4 & \text { if } i=1 \\ 2 & \text { if } i=2 \\ 5 & \text { if } i=3 \\ 4 i-6 & \text { if } 4 \leq i \leq r+1 \\ 4 n-4 i+7 & \text { if } r+2 \leq i \leq n-1 \\ 8 & \text { if } i=n\end{cases}
$$

It is clear that,

$$
\begin{aligned}
& f(E) \cup f^{v}(V) \\
&=\{1,3,7,12,16, \cdots, 4 r, 4 r-3,4 r-7, \cdots, 13,9,6\} \cup \\
&\{4,2,5,10,14, \cdots, 4 r-6,4 r-2,4 r-1,4 r-5, \cdots, 15,11,8\} \\
&=\{1,3,6,7,12,16,20, \cdots, 4 r, 9,13, \cdots, 4 r-7,4 r-3\} \cup \\
&\{2,4,5,8,10,14,18, \cdots, 4 r-6,4 r-2,11,15,19, \cdots, 4 r-5,4 r-1\} \\
&=\{1,2,3,4,5,6,7,8\} \cup\{9,13,4 r-3\} \cup\{10,14, \cdots, 4 r-2\} \\
& \cup\{11,15, \cdots, 4 r-1\} \cup\{12,16, \cdots, 4 r\} \\
&=\{1,2,3, \cdots, 4 r-3,4 r-2,4 r-1,4 r=2 n\} \\
&=\{1,2,3, \cdots, 2 n\}
\end{aligned}
$$

Hence we have proved that all cycles $C_{n}$, except $C_{4}$, are Super Vertex Mean graphs. 
Super vertex-mean labeling of $C_{9}$ and $C_{10}$ is shown in Figure 3.1.
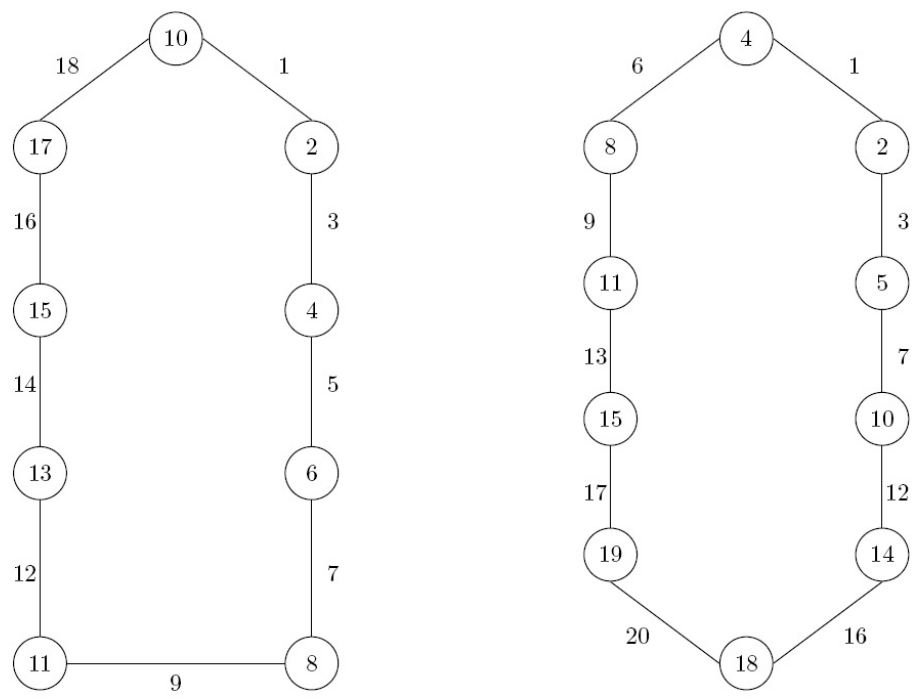

Figure 3.1

\section{4. types of svm labeling of cycles}

Any cycle $C_{n}, n \geq 3$ and $n \neq 4$ can be SVM labeled in a number of different ways. Therefore, the need arises to categorize various types of these labelings.

In Figure 4.1 we show that $C_{7}$ can be labeled in 3 different ways. 

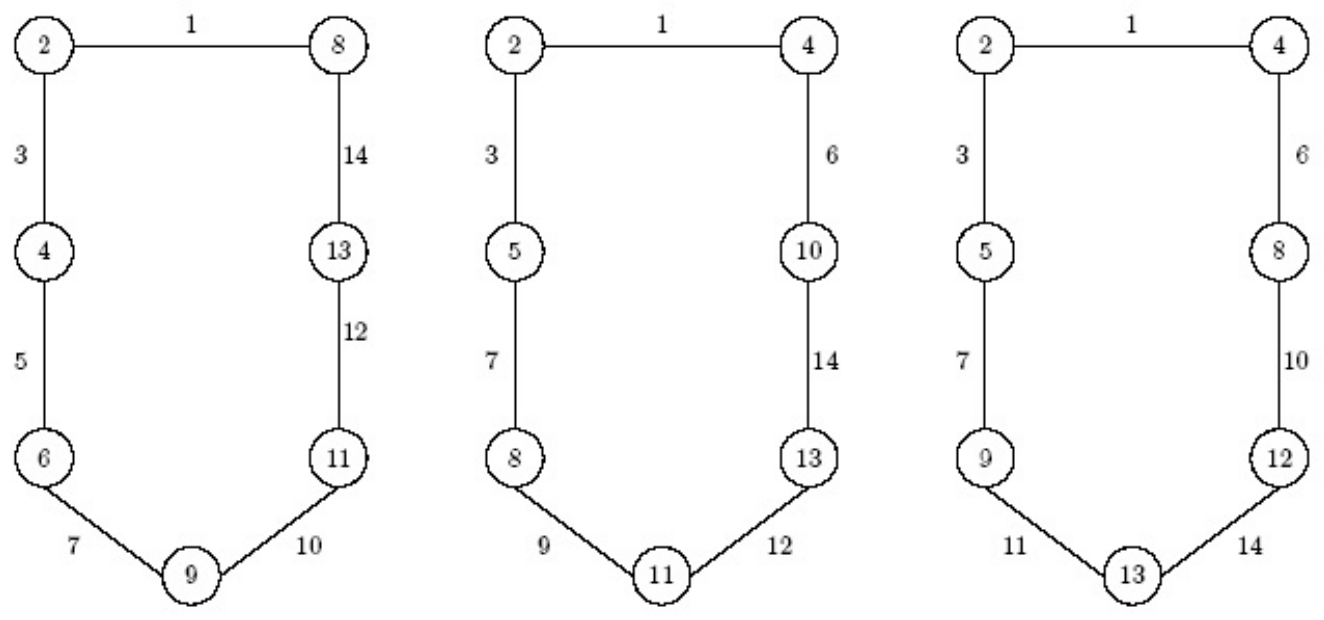

Figure4.1

Now we give $s$ - type labeling of Cycles, $C_{m}, m \geq 3$ and $m \neq 4$

Definition 4.1. We denote a super vertex mean labeling $f: E \rightarrow\{1,2, \cdots, 2 m\}$ of a cycle $C_{m}, m \geq 3$ and $m \neq 4$, that places 1 and $2 m$ on two edges such that the number of internal vertices along the shortest path connecting these two edges is $s$, as $s$-type labeling, where $1 \leq s \leq\left\lfloor\frac{m}{2}\right\rfloor$.

\section{5. $s$ - type labeling of all cycles}

In order to define completely the various types of SVM labeling of $C_{n}, n \geq 3$ and $n \neq 4$, we have two cases, based on whether $n$ is odd or even.

Case 1: $n \equiv 1(\bmod 2)$

Let $n=2 r+1,\left\{e_{1}, e_{2}, \cdots, e_{n}\right\}$ be the edge set and $\left\{v_{1}, v_{2}, \cdots, v_{n}\right\}$ be the vertex set of $C_{n}$ such that $e_{i}=v_{i} v_{i+1}, 1 \leq i \leq n-1$ and $e_{n}=v_{n} v_{1} .1$ - type labeling of cycle $C_{n}, n \geq 3$, is given as follows;

$$
f_{1}\left(e_{i}\right)= \begin{cases}2 i-1 & \text { if } 1 \leq i \leq r+1 \\ 2 i & \text { if } r+2 \leq i \leq n\end{cases}
$$

or, when we reverse the order of naming the edges and vertices, we get 
equivalently

$$
f_{1}\left(e_{i}\right)= \begin{cases}1 & \text { if } i=1 \\ 4 r-2 i+6 & \text { if } 2 \leq i \leq r+1 \\ 4 r-2 i+5 & \text { if } r+2 \leq i \leq n\end{cases}
$$

2 - type labeling, then is defined as follows for $C_{n}, n \geq 5$;

$$
f_{2}\left(e_{i}\right)= \begin{cases}1 & \text { if } i=1 \\ 4 i-2 & \text { if } i=2 \\ 4 r+4-2 i+4 & \text { if } 3 \leq i \leq r+1 \\ 4 r+4-2 i+3 & \text { if } r+2 \leq i \leq 2 r \\ 8 r-4 i+7 & \text { if } i=n\end{cases}
$$

Similarly 3 - type labeling of $C_{n}, n \geq 7$, can be defined as,

$$
f_{3}\left(e_{i}\right)= \begin{cases}1 & \text { if } i=1 \\ 4 i-2 & \text { if } 2 \leq i \leq 3 \\ 4 r+6-2 i+4 & \text { if } 4 \leq i \leq r+1 \\ 4 r+6-2 i+3 & \text { if } r+2 \leq i \leq 2 r-1 \\ 8 r-4 i+7 & \text { if } 2 r \leq i \leq n\end{cases}
$$

And when $r=s, r$ - type labeling of $C_{n}, n \geq 3$, is defined as,

$$
f_{r}\left(e_{i}\right)= \begin{cases}1 & \text { if } i=1 \\ 4 i-2 & \text { if } 2 \leq i \leq r+1 \\ 8 r-4 i+7 & \text { if } r+2 \leq i \leq n\end{cases}
$$

or, equivalently

$$
f_{r}\left(e_{i}\right)= \begin{cases}1 & \text { if } i=1 \\ 4 i-2 & \text { if } 2 \leq i \leq r=s \\ 4 r+2 r-2 i+4 & \text { if } i=r+1=s+1 \\ 4 r+2 r-2 i+3 & \text { if } i=r+2=s+2 \\ 8 r-4 i+7 & \text { if } r+3 \leq i \leq n\end{cases}
$$

Therefore, when we consider odd cycles and all types of their SVM labeling in general, we have the following theorem.

Theorem 5.1. Let $n=2 r+1,\left\{e_{1}, e_{2}, \cdots, e_{n}\right\}$ be the edge set and $\left\{v_{1}, v_{2}, \cdots, v_{n}\right\}$ be the vertex set of $C_{n}$, such that $e_{i}=v_{i} v_{i+1}, 1 \leq i \leq n-1$ and $e_{n}=v_{n} v_{1}$. Then the $s$ - type $(1 \leq s \leq r) S V M$ labeling of cycle $C_{n}, n \equiv 1(\bmod 2), n \geq$ 3 , is given as follows: 


$$
f_{s}\left(e_{i}\right)= \begin{cases}1 & \text { if } i=1 \\ 4 i-2 & \text { if } 2 \leq i \leq s \\ 4 r+2 s-2 i+4 & \text { if } s+1 \leq i \leq r+1 \\ 4 r+2 s-2 i+3 & \text { if } r+2 \leq i \leq 2 r-s+2 \\ 8 r-4 i+7 & \text { if } 2 r-s+3 \leq i \leq n\end{cases}
$$

Proof. Let $n=2 r+1$ and $n \equiv 1(\bmod 2)$. Let $\left\{e_{1}, e_{2}, \cdots, e_{n}\right\}$ be the edge set and $\left\{v_{1}, v_{2}, \cdots, v_{n}\right\}$ be the vertex set of $C_{n}$ such that $e_{i}=v_{i} v_{i+1}, 1 \leq$ $i \leq n-1$ and $e_{n}=v_{n} v_{1}$. The edges of $C_{n}$ can be s - type labeled, $1 \leq s \leq r$, as given in the Theorem 5.1. Clearly $f_{s}$ is an injective function with range from $\{1,2, \cdots, 2 n\}$.

The induced vertex labeling is given as follows:

When $s=1$,

$$
f_{1}^{v}\left(v_{i}\right)= \begin{cases}2 & \text { if } i=1 \\ n+1 & \text { if } i=2 \\ 4 r-2 i+7 & \text { if } 3 \leq i \leq r+2 \\ 4 r-2 i+6 & \text { if } r+3 \leq i \leq n\end{cases}
$$

It is evident that,

$$
\begin{aligned}
& f_{1}(E) \cup f_{1}^{v}(V) \\
&=\{1,2 n, 2 n-2,2 n-5, \cdots, n+5, n+3, n, n-2, n-4, \cdots, 5,3\} \cup \\
&\{2, n+1,2 n-1,2 n-3, \cdots, n+4, n+2, n-1, n-3, \cdots, 6,4\} \\
&=\{1,3, \cdots, n, n+3, n+5, \cdots, 2 n\} \cup \\
&\{2,4, \cdots, n-1, n+1, n+2, n+4, \cdots, 2 n-1\} \\
&=\{1,2,3, \cdots, 2 n\} . \\
& \text { When } s=r,
\end{aligned}
$$

$$
f_{r}^{v}\left(v_{i}\right)= \begin{cases}2 & \text { if } i=1 \\ 4 i-4 & \text { if } 2 \leq i \leq r+1 \\ 4 r+1=2 n-1 & \text { if } i=r+2 \\ 8 r-4 i+9 & \text { if } r+3 \leq i \leq n\end{cases}
$$

It is clear that, 


$$
\begin{aligned}
& f_{r}(E) \cup f_{r}^{v}(V) \\
&=\{1,6,10, \cdots, 2 n, 2 n-3,2 n-7, \cdots, 3\} \cup \\
&\{2,4,8, \cdots, 2 n-2,2 n-1,2 n-5, \cdots, 9,5\} \\
&=\{1,3,7, \cdots, 2 n-3,6,10, \cdots, 2 n\} \cup \\
&\{2,4,8, \cdots, 2 n-2,2 n-1,5,9, \cdots, 2 n-5\} \\
&=\{1,2,3,4,5, \cdots, 2 n-3,2 n-2,2 n-1,2 n\} .
\end{aligned}
$$

Therefore, in the more general case, the induced vertex labels are given as follows:

$$
f_{s}^{v}\left(v_{i}\right)= \begin{cases}2 & \text { if } i=1 \\ 4 i-4 & \text { if } 2 \leq i \leq s \\ 2 r+2 s & \text { if } i=s+1 \\ 4 r+2 s-2 i+5 & \text { if } s+2 \leq i \leq r+2 \\ 4 r+2 s-2 i+4 & \text { if } r+3 \leq i \leq 2 r-s+2 \\ 8 r-4 i+9 & \text { if } 2 r-s+3 \leq i \leq n\end{cases}
$$

Clearly it is injective and

$$
f_{s}(E) \cup f_{s}^{v}(V)=\{1,2,3,4,5, \cdots, 2 n-3,2 n-2,2 n-1,2 n\} .
$$

Therefore,

$$
f_{s}(E) \cup f_{s}^{v}(V)=\{1,2,3,4,5, \cdots, 2 n-3,2 n-2,2 n-1,2 n\} .
$$

Hence we have proved that all odd cycles $C_{n}$, can be s - type labeled, where $1 \leq s \leq r$ and $n=2 r+1$. Hence the proof.

Case 2: $n \equiv 0(\bmod 2)$

Let $C_{n}$ be an even cycle and $n=2 r$ where, $n \geq 6$.

Let $\left\{e_{1}, e_{2}, \cdots, e_{n}\right\}$ be the edge set and $\left\{v_{1}, v_{2}, \cdots, v_{n}\right\}$ be the vertex set of $C_{n}$ such that $e_{i}=v_{i} v_{i+1}, 1 \leq i \leq n-1$ and $e_{n}=v_{n} v_{1}$.

By checking various possibilities we find that 1 - type labeling is not possible for even cycles. So we assume that $2 \leq s \leq r$.

2 - type labeling of $C_{n}, n \geq 6$, is given as follows: 


$$
f_{2}\left(e_{i}\right)= \begin{cases}1 & \text { if } i=1 \\ 7 & \text { if } i=2 \\ 4 r-2 i+6 & \text { if } 3 \leq i \leq r \\ 4 r-2 i+5 & \text { if } r+1 \leq i \leq 2 r-2 \\ 6 & \text { if } i=2 r-1 \\ 3 & \text { if } i=2 r .\end{cases}
$$

Similarly, 3 - type labeling of $C_{n}, n \geq 8$, is as follows;

$$
f_{3}\left(e_{i}\right)= \begin{cases}1 & \text { if } i=1 \\ 7 & \text { if } i=2 \\ 12 & \text { if } i=3 \\ 4 r-2 i+8 & \text { if } 4 \leq i \leq r \\ 4 r-2 i+7 & \text { if } r+1 \leq i \leq 2 r-3 \\ 9 & \text { if } i=2 r-2 \\ 6 & \text { if } i=2 r-1 \\ 3 & \text { if } i=2 r\end{cases}
$$

And 4 - type labeling of $C_{n}, n \geq 10$, is given below;

$$
f_{4}\left(e_{i}\right)= \begin{cases}1 & \text { if } i=1 \\ 7 & \text { if } i=2 \\ 4 i & \text { if } 3 \leq i \leq 4 \\ 4 r-2 i+10 & \text { if } 5 \leq i \leq r \\ 4 r-2 i+9 & \text { if } r+1 \leq i \leq 2 r-4 \\ 8 r-4 i+1 & \text { if } 2 r-3 \leq i \leq 2 r-2 \\ 6 & \text { if } i=2 r-1 \\ 3 & \text { if } i=2 r\end{cases}
$$

and, when $s=r$, the $r$ - type labeling is given by,

$$
f_{r}\left(e_{i}\right)= \begin{cases}1 & \text { if } i=1 \\ 4 i-2 & \text { if } 2 \leq i \leq r-1 \\ r+3 i-3 & \text { if } r \leq i \leq r+1 \\ 8 r-4 i+3 & \text { if } r+2 \leq i \leq n\end{cases}
$$

As in the previous case, for all even cycles, $s$ - type labeling is defined in the following theorem: 
Theorem 5.2. Let $n=2 r$. Let $C_{n}$ be an even cycle with $n$ vertices and $\left\{e_{1}, e_{2}, \cdots, e_{n}\right\}$ be the edge set of $C_{n}$ such that $e_{i}=v_{i} v_{i+1}, 1 \leq i \leq n-1$ and $e_{n}=v_{n} v_{1}$. The $s$ - type $(2 \leq s \leq r-1) S V M$ labeling of $C_{n}, n \geq 6$, is given as follows:

$$
f_{s}\left(e_{i}\right)= \begin{cases}1 & \text { if } i=1 \\ 7 & \text { if } i=2 \\ 4 i & \text { if } 3 \leq i \leq s \\ 4 r-2 i+2 s+2 & \text { if } s+1 \leq i \leq r \\ 4 r-2 i+2 s+1 & \text { if } r+1 \leq i \leq 2 r-s \\ 8 r-4 i+1 & \text { if } 2 r-s+1 \leq i \leq 2 r-2 \\ 6 r-3 i+3 & \text { if } 2 r-1 \leq i \leq n\end{cases}
$$

and, when $s=r$, the $r$-type labeling is given by,

$$
f_{r}\left(e_{i}\right)= \begin{cases}1 & \text { if } i=1 \\ 4 i-2 & \text { if } 2 \leq i \leq r-1 \\ r+3 i-3 & \text { if } r \leq i \leq r+1 \\ 8 r-4 i+3 & \text { if } r+2 \leq i \leq n\end{cases}
$$

Proof. Let $n \equiv 0(\bmod 2)$, and $n=2 r$ and $\left\{e_{1}, e_{2}, \cdots, e_{n}\right\}$ be the edge set and $\left\{v_{1}, v_{2}, \cdots, v_{n}\right\}$ be the vertex set of $C_{n}$ such that $e_{i}=v_{i} v_{i+1}, 1 \leq$ $i \leq n-1$ and $e_{n}=v_{n} v_{1}$.

Case 1: When $2 \leq s \leq r-1$, the edges of $C_{n}, n \geq 6$, can be $s-$ type labeled as given below:

$$
f_{s}\left(e_{i}\right)= \begin{cases}1 & \text { if } i=1 \\ 7 & \text { if } i=2 \\ 4 i & \text { if } 3 \leq i \leq s \\ 4 r-2 i+2 s+2 & \text { if } s+1 \leq i \leq r \\ 4 r-2 i+2 s+1 & \text { if } r+1 \leq i \leq 2 r-s \\ 8 r-4 i+1 & \text { if } 2 r-s+1 \leq i \leq 2 r-2 \\ 6 r-3 i+3 & \text { if } 2 r-1 \leq i \leq n\end{cases}
$$

Clearly $f_{s}$ is an injective function with range from $\{1,2, \ldots, 2 n\}$. The induced vertex labeling is given as follows: 
When $s=2$

$$
f_{s}^{v}\left(v_{i}\right)= \begin{cases}2 i & \text { if } 1 \leq i \leq 2 \\ 2 r+4 & \text { if } i=3 \\ 4 r-2 i+2 s+3 & \text { if } 4 \leq i \leq r+1 \\ 4 r-2 i+2 s+2 & \text { if } r+2 \leq i \leq 2 r-2 \\ 6 r-3 i+5 & \text { if } 2 r-1 \leq i \leq n\end{cases}
$$

and when $s \geq 3$, we have

$$
f_{s}^{v}\left(v_{i}\right)= \begin{cases}2 i & \text { if } 1 \leq i \leq 2 \\ 2 i+4 & \text { if } i=3 \\ 4 i-2 & \text { if } 4 \leq i \leq s \\ 2 r+2 s & \text { if } i=s+1 \\ 4 r-2 i+2 s+3 & \text { if } s+2 \leq i \leq r+1 \\ 4 r-2 i+2 s+2 & \text { if } r+2 \leq i \leq 2 r-s \\ 4 s-1 & \text { if } i=2 r-s+1 \\ 8 r-4 i+3 & \text { if } 2 r-s+2 \leq i \leq 2 r-2 \\ 6 r-3 i+5 & \text { if } 2 r-1 \leq i \leq n\end{cases}
$$

Clearly it is an injective function and, it is also evident that, when $s=2$,

$$
\begin{aligned}
& f_{2}(E) \cup f_{2}^{v}(V) \\
&=\{1,7,4 r, 4 r-2, \cdots, 2 r+6,2 r+3,2 r+1, \cdots, 9,6,3\} \cup \\
&\{2,4,2 r+4,4 r-5,4 r-3, \cdots, 2 r+5,2 r+2, \cdots, 10,8,5\} \\
&=\{1,2,3,4,5,6,7,8,9, \cdots, 4 r-3,4 r-2,4 r-1,4 r\} .
\end{aligned}
$$

And for $3 \leq s \leq r-1$, 


$$
\begin{aligned}
f_{s}(E) \cup f_{s}^{v}(V) & \{1,7,12, \cdots, 4 s, 4 r, 4 r-2, \cdots, 2 r+2 s+2,2 r+2 s-1, \\
& 2 r+2 s-3, \cdots, 4 s+3,4 s+1,4 s-3,4 s-7, \cdots, 9,6,3\} \cup \\
& \{2,4,10,14,18, \cdots, 4 s-2,2 r+2 s, 4 r-1,4 r-3, \cdots, 2 r+2 s+1, \\
& 2 r+2 s-2,2 r+2 s-4, \cdots, 4 s+2,4 s-1,4 s-5,4 s-9, \cdots, 11,8,5\} \\
= & \{1,3,6,7,9,13, \cdots, 4 s-7,4 s-3,12,16, \cdots, 4 s, 4 r, 4 r-2, \cdots, \\
& 2 r+2 s+2,2 r+2 s-1,2 r+2 s-3, \cdots, 4 s+3,4 s+1\} \cup \\
& \{2,4,5,8,10,14, \cdots, 4 s-2,11,15, \cdots, 4 s-9,4 s-5,4 s-1,4 s+2, \\
& 4 s, \cdots, 2 r+2 s-4,2 r+2 s, 4 r-1,4 r-3, \cdots, 2 r+2 s+1\} \\
= & \{1,2,3,4,5,6,7,8,9,10, \cdots, 4 r-2,4 r-1,4 r\} .
\end{aligned}
$$

Case 2: When $s=r$, the edges of $C_{n}, n \geq 6$, can be $r$-type labeled as given below:

$$
f_{r}\left(e_{i}\right)=\left\{\begin{array}{l}
1 \\
\text { if } \quad \mathrm{i}=1 \\
4 \mathrm{i}-2 \\
\text { if } 2 \leq i \leq r-1 \\
\mathrm{r}+3 \mathrm{i}-3 \\
\text { if } \mathrm{r} \leq i \leq r+1 \\
8 \mathrm{r}-4 \mathrm{i}+3 \\
\text { if } \mathrm{r}+2 \leq i \leq n
\end{array}\right.
$$

Clearly, $f_{r}$ is an injective function with range from $\{1,2,3, \cdots, 2 n\}$. The induced vertex labeling is given as follows:

$$
f_{r}^{v}\left(v_{i}\right)= \begin{cases}2 i & \text { if } 1 \leq i \leq 2 \\ 4 i-4 & \text { if } 3 \leq i \leq r \\ 4 r-1 & \text { if } i=r+1 \\ 4 r-2 & \text { if } i=r+2 \\ 8 r-4 i+5 & \text { if } r+3 \leq i \leq n\end{cases}
$$


It is clear now that,

$$
f_{r}(E) \cup f_{r}^{v}(V)=\{1,2,3, \cdots, 2 n-2,2 n-1,2 n\} .
$$

Hence we have proved that all even cycles $C_{n}$, can be s - type, $(1 \leq s \leq$ $r, n \geq 6$ and $n=2 r)$, SVM - labeled. Thus the proof.

\section{Conclusion}

While analyzing the Super Vertex Mean labeling for cycles, $C_{n}$, we observe that the ideal situation would have been that the sum of all the edge labels to be equal to the sum of all vertex labels, as the induced vertex labels are the averages of the two edge labels of the edges that are incident on the vertex and each edge is considered twice to obtain the induced vertex labels.

But we notice that in the case of odd cycles, $C_{n}, n \equiv 1(\bmod 2)$, be it any type of SVM - labeling, there are exactly two vertices which have such edges incident on it, that are labeled with two integers one of which is odd and the other is even. Therefore, the induced vertex labels of these two vertices are 0.5 each more than the actual average of the labels of the incident edges on it, as per the definition of the SVM labeling. When we sum up all the induced vertex labels, we get an integer which is exactly one more than the sum of all the edge labels. Or in other words, this sum of all induced vertex labels is 0.5 more than the half of the sum of first $2 n$ positive integers. Similarly the sum of all edge labels is 0.5 less than the half of the sum of the first $2 n$ positive integers.

We also know that the half of the sum the of first $2 n$ positive integers is

$$
\frac{(2 n)(2 n+1)}{4}
$$

For example, 2 - type labeling of $C_{5}$, where, $2 n=10$, and

Half of the sum of first 10 positive integers $=$

$$
\frac{10 \times 11}{4}=27.5
$$

The sum of the vertex labels is $2+4+8+9+5=28$, and The sum of the edge labels is $1+6+10+7+3=27$.

Therefore, the sum of the vertex labels for $C_{n}, n \equiv 1(\bmod 2)$, is given by 
the following equation:

$$
\sum_{i=1}^{n} f^{v}\left(v_{i}\right)=\left(\frac{(2 n)(2 n+1)}{4}+0.5\right)
$$

and,

the sum of the edge labels for $C_{n}, n \equiv 1(\bmod 2)$, is

$$
\sum_{i=1}^{n} f\left(e_{i}\right)=\left(\frac{(2 n)(2 n+1)}{4}-0.5\right) .
$$

Similarly, for even cycles, $C_{n}, n \equiv 0(\bmod 2)$, there are exactly 4 vertices which have edges incident on them in such a manner that they are labeled with integers of which one is odd and the other is even, resulting in an increase of 2 in the sum of the vertex labels to that of the edge labels.

Therefore, sum of the edge labels $=$ sum of the vertex labels -2

Also, sum of the first $2 n$ positive integers $=\frac{(2 n)(2 n+1)}{2}$

So, sum of the vertex labels $=\frac{(2 n)(2 n+1)}{2}-$ sum of the edge labels

i.e., $=\frac{(2 n)(2 n+1)}{2}-$ sum of the vertex labels +2

i.e., $2 \times$ sum of the vertex labels $=\frac{(2 n)(2 n+1)}{2}+2$

Therefore, the sum of the vertex labels of $C_{n}, n \equiv 0(\bmod 2)$, is given by the following equation,

$$
\sum_{i=1}^{n} f^{v}\left(v_{i}\right)=\left(\frac{(2 n)(2 n+1)}{4}+1\right) .
$$

and sum of the edge labels for $C_{n}, n \equiv 0(\bmod 2)$ is

$$
\sum_{i=1}^{n} f\left(e_{i}\right)=\left(\frac{(2 n)(2 n+1)}{4}-1\right) .
$$

We conclude by stating that the above equations are not sufficient but necessary conditions for a set of integers from the set of first $2 n$ positive integers to be the edge label set, $f(E)$ or the induced vertex label set, $f^{v}(V)$ 
of a Super Vertex Mean labeling of any type for any cycle $C_{n}$. It is given as follows:

$$
\begin{aligned}
& \sum_{i=1}^{n} f\left(e_{i}\right)= \begin{cases}\left(\frac{(2 n)(2 n+1)}{4}-0.5\right) & \text { if } n \equiv 1(\bmod 2) \\
\left(\frac{(2 n)(2 n+1)}{4}-1\right) & \text { if } n \equiv 0(\bmod 2) .\end{cases} \\
& \sum_{i=1}^{n} f^{v}\left(v_{i}\right)= \begin{cases}\left(\frac{(2 n)(2 n+1)}{4}+0.5\right) & \text { if } n \equiv 1(\bmod 2) \\
\left(\frac{(2 n)(2 n+1)}{4}+1\right) & \text { if } n \equiv 0(\bmod 2) .\end{cases}
\end{aligned}
$$

\section{References}

[1] B. D. Acharya and K. A. Germina, Vertex-graceful Graphs, Journal of Discrete Mathematical Science and Chryptography, 13 (5), pp. 453463, (2010).

[2] J. A. Gallian, A Dynamic Survey of Graph Labeling, The Electronic Journal of Combinatorics, 16, (2013).

[3] S. W. Golomb, How to Number a Graph, Graph Theroy and Computing (Ed.R.C.Read), Academic Press, New York, pp. 23-27, (1972).

[4] R. L. Graham and N. J. A. Solane, On additive Bases and Harmonious Graphs, SIAM, J.Alg. Discrete Methods, 1, pp. 382-404, (1980).

[5] A. Lourdusamy and M. Seenivasan, Vertex-mean Graphs, International Journal of Mathematical Combinatorics, 3, pp. 114-120, (2011).

[6] A. Lourdusamy and M. Seenivasan, Mean Labelings of Cyclic Snakes, AKCE International Journal of Graphs and Combinatorics, 8 (2), pp. 105-113, (2011).

[7] A. Lourdusamy, M. Seenivasan, Sherry George and R.Revathy, Super Vertex-Mean Graphs, Sciencia Acta Xaveriana, 5 (2), pp. 39-46, (2014).

[8] R. Ponraj. Studies in Labelings of Graphs, Ph.D.thesis, Manonmaniam Sundaranar University, India, (2004).

[9] R. Ponraj and D. Ramya, On Super Mean Graphs of Order 5, Bulletin of Pure and Applied Sciences 25 (1), pp. 143-148, (2006).

[10] D. Ramya, R. Ponraj, and P. Jeyanthi, Super Mean Labeling of Graphs, Ars Combin., 112, pp. 65-72, (2013). 
[11] A. Rosa, On Certain Valuations of the Vertices of a Graph,in: Theory of Graphs (International Symposium, Rome, July 1966; Gordon and Breach, N.Y. and Dunod Paris, pp. 349-355, (1967).

[12] M. Seenivasan, Studies in Graph Theory; Some New Labeling Concepts, Ph. D. thesis, Manonmaniam Sundaranar University, India, (2013).

[13] S. Somasundaram and R. Ponraj, Super Mean Labeling of Graphs, National Academy, Science Letters, 26, pp. 210-213, (2003).

[14] R. Vasuki and A. Nagrajan, Some Results on Super Mean Labeling of Graphs, International Journal of Mathematical Combinatorics, 3, pp. 82-96, (2009).

[15] D. B. West, Introduction to Graph Theory, Prentice-Hall of India, Private Limited, New Delhi, (1996).

\author{
A. Lourdusamy \\ Department of Mathematics \\ St.Xavier's College (Autonomous), \\ Palayankottai, \\ Tirunelveli - 627002, \\ India \\ e-mail : lourdusamy15@gmail.com \\ and

\section{Sherry George} \\ Department of Mathematics \\ St.Xavier's College (Autonomous), \\ Palayankottai, \\ Tirunelveli - 627002, \\ India \\ e-mail : 1212sherry@gmail.com
}

